International Journal of Modern Physics A

(C) World Scientific Publishing Company

\title{
CASIMIR EFFECT IN DE SITTER SPACETIME
}

\author{
A. A. SAHARIAN \\ Department of Physics, Yerevan State University \\ 1 Alex Manoogian Street, 0025 Yerevan, Armenia \\ saharian@ysu.am
}

Received Day Month Year

Revised Day Month Year

\begin{abstract}
The vacuum expectation value of the energy-momentum tensor and the Casimir forces are investigated for a massive scalar field with an arbitrary curvature coupling parameter in the geometry of two parallel plates, on the background of de Sitter spacetime. The field is prepared in the Bunch-Davies vacuum state and is constrained to satisfy Robin boundary conditions on the plates. The vacuum energy-momentum tensor is non-diagonal, with the off-diagonal component corresponding to the energy flux along the direction normal to the plates. It is shown that the curvature of the background spacetime decisively influences the behavior of the Casimir forces at separations larger than the curvature radius of de Sitter spacetime. In dependence of the curvature coupling parameter and the mass of the field, two different regimes are realized, which exhibit monotonic or oscillatory behavior of the forces. The decay of the Casimir force at large plate separation is shown to be power-law, with independence of the value of the field mass.
\end{abstract}

Keywords: Casimir effect; de Sitter spacetime.

PACS numbers: 04.62.+v, 04.20.Gz, 04.50.-h, 11.10.Kk

\section{Introduction}

An interesting topic in the investigations of the Casimir effect (for a review see Refs. (1) is its explicit dependence on the geometry of background spacetime. Analytic solutions can be found for highly symmetric geometries only. In particular, motivated by Randall-Sundrum type braneworld scenarios, investigations of the Casimir effect in anti-de Sitter (AdS) spacetime have attracted a great deal of attention. The braneworld corresponds to a manifold with boundaries and all fields which propagate in the bulk give Casimir-type contributions to the vacuum energy and, as a result, to the forces acting on the branes. The Casimir effect provides in this context a natural mechanism for stabilizing the radion field.

Another popular background in cosmology is de Sitter (dS) spacetime. There are several physical motivations for this. $\mathrm{dS}$ spacetime is the maximally symmetric solution of the Einstein equations with a positive cosmological constant and due to its high symmetry numerous physical problems are exactly solvable on this background. A better understanding of physical effects in this background could serve as 
a handle to deal with more complicated geometries. In most inflationary models dS spacetime is employed to solve a number of problems in standard cosmology 2 . More recently astronomical observations of high redshift supernovae, galaxy clusters and cosmic microwave background $\frac{3}{3}$ indicate that at the present epoch the universe is accelerating and can be well approximated by a world with a positive cosmological constant. If the universe would accelerate indefinitely, the standard cosmology would lead to an asymptotic dS universe.

In the the present talk, based on Refs. 4, 5, we investigate the vacuum expectation value (VEV) of the energy-momentum tensor and the Casimir forces for a scalar field with general curvature coupling parameter in the geometry of two parallel plates on the background of $(D+1)$-dimensional dS spacetime (for previous research on the Casimir effect in dS spacetime see references given in Ref. 4). The paper is organized as follows. In the next section the VEV of the energy-momentum tensor is studied for a scalar field with general curvature coupling parameter and with Robin boundary conditions on the plates. The Casimir forces acting on the plates are discussed in section 3 . Section 4 contains a summary of the work.

\section{VEV of the energy-momentum tensor}

We consider a scalar field $\varphi(x)$ with the curvature coupling parameter $\xi$. The corresponding field equation has the form

$$
\left(\nabla_{l} \nabla^{l}+m^{2}+\xi R\right) \varphi=0
$$

where $\nabla_{l}$ is the covariant derivative operator and $R$ is the Ricci scalar for the background spacetime. The values of the curvature coupling parameter $\xi=0$ and $\xi=\xi_{D} \equiv(D-1) / 4 D$, with $D$ being the number of spatial dimensions, correspond to the most important special cases of minimally and conformally coupled fields. In the present paper the background geometry is the $(D+1)$-dimensional dS spacetime. We write the corresponding line element in planar coordinates, most appropriate for cosmological applications:

$$
d s^{2}=d t^{2}-e^{2 t / \alpha} \sum_{i=1}^{D}\left(d z^{i}\right)^{2} .
$$

The Ricci scalar is related to the parameter $\alpha$ in the scale factor by the formula $R=D(D+1) / \alpha^{2}$. In addition to the synchronous time coordinate $t$, we will use the conformal time $\tau$ defined by the relation $\tau=-\alpha e^{-t / \alpha},-\infty<\tau<0$.

Our main interest are the VEV of the energy-momentum tensor and the Casimir forces in the geometry of two infinite, parallel plates located at $z^{D}=a_{j}, j=1,2$. On the plates the field obeys Robin boundary conditions (BCs)

$$
\left(1+\beta_{j} n^{l} \nabla_{l}\right) \varphi(x)=0, \quad z^{D}=a_{j},
$$

with constant coefficients $\beta_{j}$ and with $n^{l}$ being the normal to the boundary. For the region between the plates one has $n^{l}=(-1)^{j-1} \delta_{D}^{l}$. Dirichlet and Neumann BCs 
correspond to special cases $\beta_{j}=0$ and $\beta_{j}=\infty$, respectively. The imposition of BCs leads to a modification of the VEVs for physical quantities, as compared with those in the situation without boundaries. Among the most important characteristics of the vacuum state is the VEV of the energy-momentum tensor. In addition to describing the physical structure of the quantum field at a given point, the energymomentum tensor acts as the source in the Einstein equations and therefore plays an important role in modelling a self-consistent dynamics involving the gravitational field. The VEV is expressed as the mode-sum

$$
\left\langle 0\left|T_{i k}\right| 0\right\rangle=\sum_{\sigma} T_{i k}\left\{\varphi_{\sigma}(x), \varphi_{\sigma}^{*}(x)\right\},
$$

where $\left\{\varphi_{\sigma}(x), \varphi_{\sigma}^{*}(x)\right\}$ is a complete set of solutions to the classical field equation satisfying the boundary conditions, and the bilinear form $T_{i k}\{\varphi(x), \psi(x)\}$ is determined by the classical energy-momentum tensor. We implicitly assume the presence of a cutoff function in (4) which makes the sum finite.

In the problem under consideration, the time-dependent part of the eigenfunctions is a linear combination of the functions $\eta^{D / 2} H_{\nu}^{(l)}(\eta K), l=1,2$, where $H_{\nu}^{(l)}(x)$ is the Hankel function, $\eta=|\tau|$, and

$$
\nu=\left[D^{2} / 4-D(D+1) \xi-m^{2} \alpha^{2}\right]^{1 / 2} .
$$

Different choices of the coefficients in this linear combination correspond to different choices of the vacuum state. We will consider dS invariant Bunch-Davies vacuum $\underline{6}$ for which the coefficient for the part containing the function $H_{\nu}^{(2)}(\eta K)$ is zero. In the region between the plates, $a_{1}<z^{D}<a_{2}$, the corresponding eigenfunctions, satisfying the $\mathrm{BC}$ on the plate at $z^{D}=a_{1}$, have the form

$$
\varphi_{\sigma}(x)=C_{\sigma} \eta^{D / 2} H_{\nu}^{(1)}(\eta K) \cos \left[k_{D}\left(z^{D}-a_{1}\right)+\alpha_{1}\left(k_{D}\right)\right] e^{i \mathbf{k} \cdot \mathbf{z}},
$$

with the notations $K=\sqrt{k^{2}+k_{D}^{2}}$ and $e^{2 i \alpha_{1}(x)}=\left(i \beta_{1} x-1\right) /\left(i \beta_{1} x+1\right)$. In Eq. (6) , $\mathbf{z}=\left(z^{1}, \ldots, z^{D-1}\right)$ is the position vector along the dimensions parallel to the plates and $\mathbf{k}=\left(k_{1}, \ldots, k_{D-1}\right)$. For a conformally coupled massless field one has $\nu=1 / 2$. From the boundary condition on the plate $z^{D}=a_{2}$ it follows that the eigenvalues for $k_{D}$ are solutions of the equation

$$
\left(1-b_{1} b_{2} y^{2}\right) \sin y-\left(b_{1}+b_{2}\right) y \cos y=0, y=k_{D} a,
$$

where $b_{j}=\beta_{j} / a$ and $a=a_{2}-a_{1}$. In the discussion below we will assume that all zeros are real. In particular, this is the case for the conditions $b_{j} \leqslant 0$ (see Ref. 7). The positive solutions of Eq. (77) will be denoted by $y=\lambda_{n}, n=1,2, \ldots$, and for the eigenvalues of $k_{D}$ one has $k_{D}=\lambda_{n} / a$. Consequently, the eigenfunctions are specified by the set $\sigma=(\mathbf{k}, n)$. The coefficient $C_{\sigma}$ in (6) is determined from the normalization condition and is given by the expression

$$
C_{\sigma}^{2}=\frac{(2 \pi)^{2-D} \alpha^{1-D} e^{i\left(\nu-\nu^{*}\right) \pi / 2}}{4 a\left\{1+\cos \left[\lambda_{n}+2 \alpha_{1}\left(\lambda_{n} / a\right)\right] \sin \left(\lambda_{n}\right) / \lambda_{n}\right\}},
$$

the star meaning complex conjugate. 
It is well known that in dS spacetime without boundaries the Bunch-Davies vacuum state is not a physically realizable state for $\operatorname{Re} \nu \geqslant D / 2$. The corresponding two-point functions contain infrared divergences. In the presence of boundaries, the BCs on the quantized field may exclude long wavelength modes and the BunchDavies vacuum becomes a realizable state. An example of this type of situation is provided by the geometry of two parallel plates described above. In the region between the plates and for BCs with $\beta_{j} \leqslant 0, \beta_{j} \neq \infty$, there is a maximum wavelength, $2 \pi a / \lambda_{1}$, and the two-point functions contain no infrared divergences. Mathematically, this situation corresponds to the one where in the argument of the Hankel function we have $K \geqslant \lambda_{1} / a$.

Combining Eqs. (4), ([6), (8), for the VEV of the energy-momentum tensor in the region between the plates we find the expression which contains series over $\lambda_{n}$. For the summation of this series we apply the Abel-Plana type summation formula from Refs. 7, 8, This allows us to write the diagonal components in the decomposed form (no summation over $l$ )

$$
\begin{aligned}
\left\langle T_{l}^{l}\right\rangle= & \left\langle T_{l}^{l}\right\rangle_{j}+\frac{A_{D}}{\alpha^{D+1}} \int_{0}^{\infty} d y y^{1-D} \int_{y}^{\infty} d x H(x, y) \\
& \times\left[g\left(\beta_{j} x / \eta,\left|z^{D}-a_{j}\right| x / \eta\right) G_{l}(y)+2 G_{l} x^{2} F_{\nu}(y)\right],
\end{aligned}
$$

where

$$
\begin{aligned}
& A_{D}=\frac{4(4 \pi)^{-(D+1) / 2}}{\Gamma((D-1) / 2)}, H(x, y)=\frac{\left(x^{2}-y^{2}\right)^{(D-3) / 2}}{c_{1}(x / \eta) c_{2}(x / \eta) e^{2 a x / \eta}-1} \\
& g\left(\beta_{j} u, y u\right)=c_{j}(u) e^{2 u y}+e^{-2 u y} / c_{j}(u)+2, c_{j}(u)=\frac{\beta_{j} u-1}{\beta_{j} u+1},
\end{aligned}
$$

and

$$
F_{\nu}(y)=y^{D}\left[I_{\nu}(y)+I_{-\nu}(y)\right] K_{\nu}(y),
$$

with $I_{\nu}(y)$ and $K_{\nu}(y)$ being the modified Bessel functions. In (9), we have introduced the notations

$$
\begin{aligned}
G_{0}(y) & =\left[\frac{y^{2}}{4} \partial_{y}^{2}-D\left(\xi+\xi_{D}\right) y \partial_{y}+D^{2} \xi+m^{2} \alpha^{2}-y^{2}+(1-4 \xi) x^{2}\right] F_{\nu}(y), \\
G_{D}(y) & =\left\{\left(\xi-\frac{1}{4}\right) y^{2} \partial_{y}^{2}+\left[\xi(2-D)+\frac{D-1}{4}\right] y \partial_{y}-\xi D\right\} F_{\nu}(y), \\
G_{l}(y) & =G_{D}(y)+\left[\frac{y^{2}-x^{2}}{D-1}+(1-4 \xi) x^{2}\right] F_{\nu}(y), l=1, \ldots, D-1,
\end{aligned}
$$

and $G_{D}=1, G_{l}=4 \xi-1$ for $l=0,1, \ldots, D-1$. In Eq. (9) (no summation over $l$ ),

$$
\left\langle T_{l}^{l}\right\rangle_{j}=\left\langle T_{l}^{l}\right\rangle_{\mathrm{dS}}+\frac{A_{D}}{\alpha^{D+1}} \int_{0}^{\infty} d y y^{1-D} \int_{y}^{\infty} d x\left(x^{2}-y^{2}\right)^{\frac{D-3}{2}} \frac{e^{-2 x\left|z^{D}-a_{j}\right| / \eta}}{c_{j}(x / \eta)} G_{l}(y),
$$

is the $\mathrm{VEV}$ for the geometry of a single plate at $z^{D}=a_{j}$ when the second plate is absent ${ }^{4}$ and $\left\langle T_{l}^{l}\right\rangle_{\mathrm{dS}}$ is the corresponding renormalized VEV in dS spacetime without 
boundaries. For points away from the plates, renormalization is required for the latter part only. Due to the dS invariance of the Bunch-Davies vacuum, $\left\langle T_{k}^{l}\right\rangle_{\mathrm{dS}}$ is proportional to the metric tensor with a constant coefficient and has been well investigated in the literature 69 . The last term on the right hand side of Eq. (9) is induced by the presence of the second plate. Note that in the formulas given above, $\left|z^{D}-a_{j}\right| / \eta$ is the proper distance of the observation point from the plate at $z^{D}=a_{j}$, measured in units of the $\mathrm{dS}$ curvature radius $\alpha$. The VEVs depend on time through the combinations $\left|z^{D}-a_{j}\right| / \eta$ and $\beta_{j} / \eta$. This property is a consequence of the maximal symmetry of dS spacetime and of Bunch-Davies vacuum.

For the non-zero off-diagonal component, we have

$$
\begin{aligned}
\left\langle T_{0}^{D}\right\rangle= & \left\langle T_{0}^{D}\right\rangle_{j}-\operatorname{sgn}\left(z^{D}-a_{j}\right) \frac{A_{D}}{2 \alpha^{D+1}} \int_{0}^{\infty} d y y^{1-D} G_{0 D}(y) \\
& \times \int_{y}^{\infty} d x x H(x, y)\left[c_{j}(x / \eta) e^{2 x\left|z^{D}-a_{j}\right| / \eta}-e^{-2 x\left|z^{D}-a_{j}\right| / \eta} / c_{j}(x / \eta)\right],
\end{aligned}
$$

where the part corresponding to the geometry of a single plate is given by

$$
\begin{aligned}
\left\langle T_{0}^{D}\right\rangle_{j}= & \operatorname{sgn}\left(z^{D}-a_{j}\right) \frac{2 A_{D}}{\alpha^{D+1}} \int_{0}^{\infty} d y y^{1-D} G_{0 D}(y) \\
& \times \int_{y}^{\infty} d x x\left(x^{2}-y^{2}\right)^{\frac{D-3}{2}} \frac{e^{-2 x\left|z^{D}-a_{j}\right| / \eta}}{c_{j}(x / \eta)} .
\end{aligned}
$$

In these formulas we have defined the function

$$
G_{0 D}(y)=\left[(4 \xi-1) y \partial_{y}+4 \xi\right] F_{\nu}(y) .
$$

The off-diagonal component (14) corresponds to the energy flux along the direction perpendicular to the plates. Depending on the values of the coefficients in the boundary conditions and of the field mass this flux can be positive or negative. In the case when $\beta_{1}=\beta_{2}$, the off-diagonal component $\left\langle T_{0}^{D}\right\rangle$ vanishes at $z^{D}=\left(a_{1}+a_{2}\right) / 2$. This property is a direct consequence of the problem symmetry.

For a conformally coupled massless scalar field $\left(\xi=\xi_{D}, m=0\right)$ the single plate part in the VEV of the energy-momentum tensor vanishes and one finds (no summation over $l$ )

$$
\left\langle T_{k}^{l}\right\rangle=\left\langle T_{k}^{l}\right\rangle_{\mathrm{dS}}-\frac{(\eta / \alpha)^{D+1} B_{l} \delta_{l}^{k}}{(4 \pi)^{D / 2} \Gamma(D / 2+1)} \int_{0}^{\infty} d x \frac{x^{D}}{c_{1}(x) c_{2}(x) e^{2 a x}-1},
$$

where $B_{l}=1$ for $l=0, \ldots, D-1$ and $B_{D}=-D$. The boundary induced part in this formula could have been obtained from the corresponding result for the Casimir effect in Minkowski spacetime, by using the fact that the two problems are conformally related (see also Refs. 10, 11 for the Casimir densities in AdS and Robertson-Walker spacetimes). Note that the boundary induced part in Eq. (17) is traceless and the trace anomaly is contained in the boundary-free part only.

In the region $z^{D}<a_{1}\left(z^{D}>a_{2}\right)$ the VEV of the energy-momentum tensor coincides with the corresponding VEV for a single plate located at $z^{D}=a_{1}\left(z^{D}=\right.$ $\left.a_{2}\right)$ and is given by the expressions (13) and (15), with $j=1(j=2)$. The results 
obtained in the present paper can be applied to a more general problem where the cosmological constant is different in separate regions $z^{D}<a_{1}, a_{1}<z^{D}<a_{2}$, and $z^{D}>a_{2}$. In this case the plate can be considered as a simple model of a thin domain wall separating the regions with different dS vacua.

In the discussion above we have considered the VEV of the bulk energymomentum tensor. For scalar fields with general curvature coupling parameter and with Robin BCs, in Ref. 7 it has been shown that in the discussion of the relation between the mode sum energy and the volume integral of the renormalized energy density for the Robin parallel plates geometry in Minkowski spacetime it is necessary to include in the energy a surface term concentrated on the boundary. An expression for the surface energy-momentum tensor for a scalar field with a general curvature coupling parameter in the general case of bulk and boundary geometries is derived in Ref. 12. The investigation of the VEV for the surface densities in the problem under consideration will be reported in Ref. 13 .

\section{Casimir forces}

Having the VEV of the energy-momentum tensor, we can evaluate the forces acting on the plates. The vacuum force acting per unit surface of the plate at $z^{D}=a_{j}$ is determined by the ${ }_{D}^{D}$-component of the vacuum energy-momentum tensor evaluated at this point. For the region between the plates, the corresponding effective pressures can be written as $p^{(j)}=p_{1}^{(j)}+p_{(\mathrm{int})}^{(j)}, j=1,2$. The term $p_{1}^{(j)}$ is the pressure for a single plate at $z^{D}=a_{j}$, when the second plate is absent. This term is divergent due to the surface divergences in the subtracted VEVs and needs additional renormalization. The term $p_{(\text {int })}^{(j)}$ is the pressure induced by the second plate, and can be termed as an interaction force. This contribution is finite for all nonzero distances between the plates. In the regions $z^{D}<a_{1}$ and $z^{D}>a_{2}$ we have $p^{(j)}=p_{1}^{(j)}$. As a result, the contributions to the vacuum force coming from the term $p_{1}^{(j)}$ are the same from the left and from the right sides of the plate, so that there is no net contribution to the effective force.

The interaction force on the plate at $z^{D}=a_{j}$ is obtained from the last term on the right hand side of Eq. (9) for $\left\langle T_{D}^{D}\right\rangle$ (with minus sign) taking $z^{D}=a_{j}$ :

$$
p_{(\mathrm{int})}^{(j)}=-\frac{2 A_{D}}{\alpha^{D+1}} \int_{0}^{\infty} d y y^{1-D} \int_{y}^{\infty} d x x^{2} H(x, y)\left[\frac{2\left(\beta_{j} / \eta\right)^{2} G_{D}(y)}{\left(\beta_{j} x / \eta\right)^{2}-1}+F_{\nu}(y)\right]
$$

where $H(x, y)$ is defined by Eq. (10). The time dependence of the forces appears in the form $a / \eta$ and $\beta_{j} / \eta$. Note that the ratio $a / \eta$ is the proper distance between the plates measured in units of dS curvature radius $\alpha$. The effective pressures (18) can be either positive or negative, leading to repulsive or to attractive forces, respectively. For $\beta_{1} \neq \beta_{2}$ the Casimir forces acting on the left and on the right plates are different. For large values of $\alpha$, to leading order, the corresponding result for the geometry of 
two parallel plates in Minkowski spacetime is obtained:

$$
p_{(\mathrm{int})}^{(j)} \approx p_{(\mathrm{M})}^{(j)}=-\frac{2(4 \pi)^{-D / 2}}{\Gamma(D / 2)} \int_{m}^{\infty} d x \frac{x^{2}\left(x^{2}-m^{2}\right)^{D / 2-1}}{c_{1}(x) c_{2}(x) e^{2 a x}-1} .
$$

Note that in the Minkowski spacetime the force is the same for both plates with independence of the values for the coefficients $\beta_{j}$ and this force does not depend on the curvature coupling parameter (for the interaction forces between the Robin plates in the geometry with an arbitrary internal space see Ref. 14).

In the special cases of Dirichlet and of Neumann boundary conditions one finds:

$$
\begin{aligned}
& p_{\text {(int) }}^{(\mathrm{D})}=-\frac{4 \alpha^{-D-1}}{(2 \pi)^{\frac{D}{2}+1}} \sum_{n=1}^{\infty} \int_{0}^{\infty} d y y F_{\nu}(y)\left[(D-1) f_{\frac{D}{2}}\left(y u_{n}\right)+f_{\frac{D}{2}-1}\left(y u_{n}\right)\right], \\
& p_{\text {(int) }}^{(\mathrm{N})}=p_{\text {(int) }}^{(\mathrm{D})}-\frac{8 \alpha^{-D-1}}{(2 \pi)^{\frac{D}{2}+1}} \sum_{n=1}^{\infty} \int_{0}^{\infty} d y \frac{G_{D}(y)}{y} f_{\frac{D}{2}-1}\left(y u_{n}\right), u_{n}=2 n a / \eta,
\end{aligned}
$$

where $f_{\mu}(x)=K_{\mu}(x) / x^{\mu}$. For $0 \leqslant \nu<1$ the integrand in the expression for $p_{(\mathrm{int})}^{(\mathrm{D})}$ is positive which corresponds to an attractive force for all separations.

Now we turn to the investigation of the asymptotic behavior for the vacuum forces in the general case of Robin BC. In the limit of small proper distances between the plates, $a / \eta \ll 1$, to leading order we find

$$
p_{(\mathrm{int})}^{(j)} \approx-\frac{2(\eta / \alpha)^{D+1}}{(4 \pi)^{D / 2} \Gamma(D / 2)} \int_{0}^{\infty} d x \frac{x^{D}}{c_{1}(x) c_{2}(x) e^{2 a x}-1} .
$$

If, in addition, $\left|\beta_{j}\right| / a \gg 1$, one has

$$
p_{(\mathrm{int})}^{(j)} \approx-\frac{D \Gamma((D+1) / 2) \zeta_{\mathrm{R}}(D+1)}{(4 \pi)^{(D+1) / 2}(\alpha a / \eta)^{D+1}},
$$

and the corresponding force is attractive. In (23), $\zeta_{\mathrm{R}}(x)$ is the Riemann zeta function. The same result is obtained for Dirichlet BCs on both plates. In the case of Dirichlet $\mathrm{BC}$ on one plate and non-Dirichlet one on the other, the leading term is obtained from Eq. (23) with an additional factor $\left(2^{-D}-1\right)$. In this case the vacuum force is repulsive at small distances.

In considering the large distance asymptotics, corresponding to $a / \eta \gg 1$, the cases of real and imaginary $\nu$ must be studied separately. For positive values of $\nu$, one has

$$
p_{\text {(int) }}^{(j)} \approx-\frac{2 \alpha^{-D-1} g_{\nu}^{(j)} \Gamma(\nu)}{\pi^{D / 2+1}(2 a / \eta)^{D-2 \nu+2}}
$$

for non-Neumann BCs on the plate at $z^{D}=a_{j}\left(\left|\beta_{j}\right|<\infty\right)$ and

$$
p_{(\mathrm{int})}^{(j)} \approx-\frac{\alpha^{-D-1} g_{\nu}^{\mathrm{N}(j)} \Gamma(\nu)}{\pi^{D / 2+1}(2 a / \eta)^{D-2 \nu}}
$$


for Neumann $\mathrm{BC}\left(\beta_{j}=\infty\right)$. Here the notations are as follows:

$$
\begin{aligned}
g_{\nu}^{(j)} & =\left(\frac{D+1}{2}-\nu\right) \Gamma(D / 2-\nu+1)\left[1-2\left(\frac{\beta_{j}}{\eta}\right)^{2} f_{D}\right] \sum_{n=1}^{\infty} \frac{\left(\delta_{1} \delta_{2}\right)^{n}}{n^{D-2 \nu+2}}, \\
g_{\nu}^{\mathrm{N}(j)} & =\Gamma(D / 2-\nu) f_{D} \sum_{n=1}^{\infty} \frac{\left(\delta_{1} \delta_{2}\right)^{n}}{n^{D-2 \nu}},
\end{aligned}
$$

with $f_{D}=-2 \nu[\xi+(\xi-1 / 4)(D-2 \nu)]$ and $\delta_{j}=c_{j}(0)$. Note that $\delta_{j}=-1$ for nonNeumann BC, while $\delta_{j}=1$ if the BC is Neumann. In the case of non-Neumann $\mathrm{BCs}$ we have assumed that $\left|\beta_{j}\right| / a \ll 1$.

As it is seen from (25), for positive values of $\nu$ and when $f_{D} \neq 0$, at large distances the ratio of the Casimir forces acting on the plate with Neumann and nonNeumann BCs is of the order $(a / \eta)^{2}$. Note that in neither of these cases does the force depend on the specific value of Robin coefficient in the $\mathrm{BC}$ on the second plate. For Dirichlet $\mathrm{BC}$ on the plate at $z^{D}=a_{j}\left(\beta_{j}=0\right)$, at large separations the Casimir force acting on that plate is repulsive (attractive) for Neumann (non-Neumann) BCs on the other plate. The nature of the force acting on the plate with Neumann BC depends on the sign of $f_{D}$ and can be either repulsive or attractive, in function of the curvature coupling parameter and of the field mass. For minimally and conformally coupled massive scalar fields one has $f_{D}=\nu(D / 2-\nu)$ and $f_{D}=\nu(1 / 2-\nu) / D$, respectively, and this parameter is positive. The corresponding force is attractive (repulsive) for Neumann (non-Neumann) BC on the second plate. Note that for the geometry of parallel plates in the Minkowski bulk the Casimir forces at large distances are repulsive for Neumann BC on one plate and for non-Neumann BC on the other plate. For all other cases of BCs the forces are attractive.

For imaginary $\nu$, the leading order terms at large separations between the plates are in the form

$$
\begin{aligned}
& p_{(\mathrm{int})}^{(j)} \approx-\frac{4 \alpha^{-D-1}\left|g_{\nu}^{(j)}\right|}{\pi^{D / 2+1}(2 a / \eta)^{D+2}} \cos \left[2|\nu| \ln (2 a / \eta)+\phi_{(j)}\right],\left|\beta_{j}\right|<\infty, \\
& p_{(\mathrm{int})}^{(j)} \approx-\frac{2 \alpha^{-D-1}\left|g_{\nu}^{\mathrm{N}(j)}\right|}{\pi^{D / 2+1}(2 a / \eta)^{D}} \cos \left[2|\nu| \ln (2 a / \eta)+\phi_{(j)}^{\mathrm{N}}\right], \beta_{j}=\infty,
\end{aligned}
$$

where the phases are defined in accordance with $g_{\nu}^{(j)}=\left|g_{\nu}^{(j)}\right| e^{i \phi_{(j)}}$ and $g_{\nu}^{\mathrm{N}(j)}=$ $\left|g_{\nu}^{\mathrm{N}(j)}\right| e^{i \phi_{(j)}^{\mathrm{N}}}$. In this case the decay of the vacuum forces is oscillatory.

Having in mind that spectral properties of spin 2, 1, 0 operators for dS spacetime are known, the current study can be extended to the calculation of the Casimir force due to quantum gravity (for the one-loop effective action of arbitrary quantum gravity in dS spacetime see Ref. 15). Note that the calculations can be extended to a self-interacting scalar field theory too, and the results described here can be used to study the curvature-induced phase transitions of the in-in effective potential in the same way as it was proposed for the out-in effective potential in Ref. 16.

In Fig. 1, we have plotted the Casimir force for a $D=3$ scalar field with Dirichlet $\mathrm{BC}$, minimally coupled to gravity, as a function of the proper distance between the 
plates, measured in units of the dS curvature scale $\alpha$. The figures near the curves correspond to the values of the parameter $m \alpha$. Values are taken in a way so to have both possibilities, with positive and purely imaginary values of the parameter $\nu$.
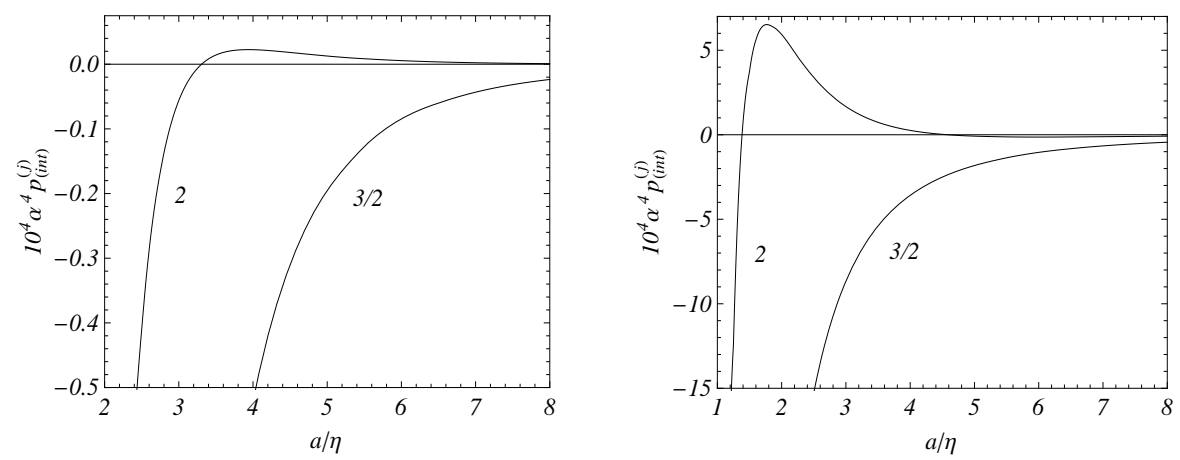

Fig. 1. Interaction forces between the plates for a $D=3$ minimally coupled scalar field with Dirichlet (left plot) and Neumann (right plot) BCs. The figures near the curves are the values of the parameter $\alpha m$.

In Fig. 2 the dependence of the Casimir force on the parameter $m \alpha$ is depicted for a given separation corresponding to $a / \eta=4$. Conformally coupled scalar fields with Dirichlet and Neumann BCs are considered. For a massless field the force is the same for Dirichlet and Neumann BCs.

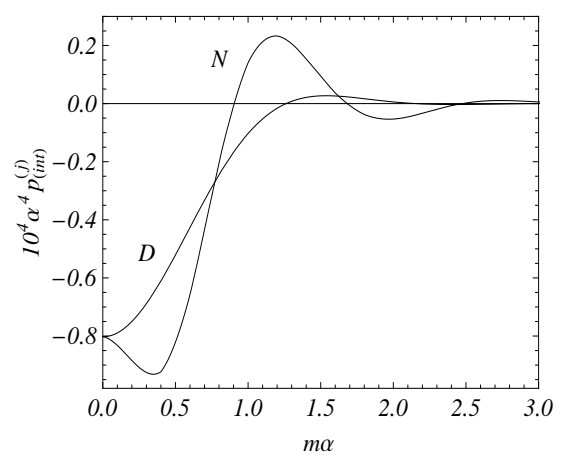

Fig. 2. Interaction force between the plates for $a / \eta=4$ as a function of the field mass, for a $D=3$ conformally coupled scalar field with Dirichlet and Neumann BCs.

From the discussion given above it follows that for proper distances between the plates larger than the curvature radius of the $\mathrm{dS}$ spacetime, $\alpha a / \eta \gtrsim \alpha$, the gravitational field essentially changes the behavior of the Casimir forces compared with the case of the plates in Minkowski spacetime. The forces may become repulsive at large separations between the plates. In particular, for real values $\nu$ and for Neumann 
$\mathrm{BC}$ on both plates, Casimir forces are repulsive at large separations, in the range of parameters for which $f_{D}<0$. Recall that, for the geometry of parallel plates on the background of Minkowski spacetime, the only case with repulsive Casimir forces at large distances corresponds to Neumann BC on one plate and non-Neumann $\mathrm{BC}$ on the other. A remarkable feature of the influence of the gravitational field is the oscillatory behavior of the Casimir forces at large distances, which appears in the case of imaginary $\nu$. In this case, the values of the plate distance yielding zero Casimir force correspond to equilibrium positions. Among them, the positions with negative derivative of the force with respect to the distance are locally stable. As it follows from asymptotic formulas (24), (25), and (27), at large separations between the plates the decay of the Casimir forces as functions of the distance is power-law for both cases of massive and massless fields. Recall that, in Minkowski spacetime the corresponding Casimir forces decay as $1 / a^{D+1}$ for a massless field and they are exponentially suppressed by the factor $\exp (-2 m a)$ for a massive filed.

It is also of interest to compare the features for the Casimir force in dS spacetime with the behavior of the Casimir forces for parallel plates in AdS spacetime. In Poincaré coordinates the corresponding line element is given by the expression

$$
d s_{\mathrm{AdS}}^{2}=e^{-\lambda y} \eta_{i k} d x^{i} d x^{k}-d y^{2},
$$

where $\eta_{i k}=\operatorname{diag}(1,-1, \ldots,-1)$ is the metric tensor for $D$-dimensional Minkowski spacetime. For the corresponding Ricci scalar one has $R=-D(D+1) \lambda^{2}$ and the AdS curvature radius is given by $1 / \lambda$. For the general case of Robin BCs on two parallel plates, located at $y=y_{j}, j=1,2$, the interaction forces between the plates are investigated in Ref. 17 (see also Refs. 18 for the case where an extra compact subspace is present). At large distances between the plates, as compared with the AdS curvature radius, $\lambda\left(y_{2}-y_{1}\right) \gg 1$, the vacuum interaction forces per unit surface, $p_{\text {(int) }}^{(j)}$, are exponentially suppressed by the factor $\exp \left[2 \nu_{\mathrm{AdS}} \lambda\left(y_{1}-y_{2}\right)\right]$ for the plate at $y=y_{1}$ and by the factor $\exp \left[\left(2 \nu_{\mathrm{AdS}}+D\right) \lambda\left(y_{1}-y_{2}\right)\right]$ for the plate at $y=y_{2}$, where

$\nu_{\text {AdS }}=\left[D^{2} / 4-D(D+1) \xi+m^{2} / \lambda^{2}\right]^{1 / 2}$. Note that in AdS spacetime the ground state becomes unstable for imaginary values of $\nu_{\text {AdS }} 19$. Hence, in AdS spacetime the Casimir forces are exponentially suppressed for both massive and massless fields.

\section{Conclusion}

The natural appearance of dS spacetime in a variety of situations has stimulated considerable interest in the behavior of quantum fields propagating in this background. In the present paper we have studied the VEV of the energy-momentum tensor and the Casimir forces for a scalar field with an arbitrary curvature coupling parameter satisfying Robin BCs on two parallel plates in dS spacetime. In the region between the plates, the VEVs are decomposed into a boundary-free dS, a single plate-induced and an interference contributions, respectively. The vacuum energymomentum tensor is non-diagonal, with the off-diagonal component corresponding to the energy flux along the direction normal to the plates. Depending on the values 
of the coefficients in the boundary conditions and of the field mass this flux can be positive or negative. In the case of a conformally coupled massless field, the single plate contribution to the VEV of the energy-momentum tensor vanishes and the interference part is obtained from the corresponding result for the Minkowski bulk, by standard conformal transformation.

The vacuum forces acting on the plates are determined by the ${ }_{D}^{D}$-component of the stress. The normal stresses on the plates are presented as sums of single plate and interaction contributions. The contributions to the vacuum force coming from the single plate terms are the same from the left and from the right sides of the plate and thus give no contribution to the effective force. The interaction forces per unit surface are determined by formula (18) for general Robin BCs and by Eqs. (20), (21) in the special cases of Dirichlet and Neumann BCs. At small distances between the plates the vacuum forces are attractive, except for the case of Dirichlet BC on one plate and non-Dirichlet on the other, in which case the force turns out to be repulsive. At large separations and for positive values of $\nu$, the force acting on the plate decays monotonically as $1 /(2 a / \eta)^{D-2 \nu+2}$, for non-Neumann BCs, and as $1 /(2 a / \eta)^{D-2 \nu}$, in the case of Neumann BCs [see Eqs. (25)]. For imaginary values of $\nu$ the behavior of the vacuum forces is damping oscillatory, in the leading order described by Eqs. (27).

From the analysis carried out above, it follows that the curvature of the background spacetime decisively influences the behavior of the Casimir forces at distances larger than the curvature scale. As we have seen, in dS spacetime the decay of the forces at large separations between the plates is power-law. This is quite remarkable and clearly in contrast with the corresponding features of the same problem in Minkowski and AdS spacetimes.

\section{Acknowledgments}

The author acknowledges the Organizers of the 8th Alexander Friedmann International Seminar on Gravitation and Cosmology and CAPES (Brazil) for a support.

\section{References}

1. E. Elizalde, S. D. Odintsov, A. Romeo, A. A. Bytsenko and S. Zerbini, Zeta Regularization Techniques with Applications (World Scientific, Singapore, 1994); V. M. Mostepanenko and N. N. Trunov, The Casimir Effect and Its Applications (Clarendon, Oxford, 1997); K. A. Milton, The Casimir Effect: Physical Manifestation of Zero-Point Energy (World Scientific, Singapore, 2002); M. Bordag, G. L. Klimchitskaya, U. Mohidden and V. M. Mostepanenko, Advances in the Casimir Effect (Oxford University Press, Oxford, 2009); G. L. Klimchitskaya, U. Mohidden and V. M. Mostepanenko, Rev. Mod. Phys. 81, 1827 (2009).

2. A. D. Linde, Particle Physics and Inflationary Cosmology (Harwood Academic Publishers, Chur, Switzerland 1990).

3. A. G. Riess et al., Astrophys. J. 659, 98 (2007); D. N. Spergel et al., Astrophys. J. Suppl. Ser. 170, 377 (2007); U. Seljak, A. Slosar and P. McDonald, J. Cosmol. Astropart. Phys. 10 (2006) 014. 
4. A. A. Saharian and T. A. Vardanyan, Class. Quantum Grav. 26, 195004 (2009).

5. E. Elizalde, A. A. Saharian and T. A. Vardanyan, Phys. Rev. D 81, 124003 (2010).

6. T. S. Bunch and P. C. W. Davies, Proc. R. Soc. London A 360, 117 (1978).

7. A. Romeo and A. A. Saharian, J. Phys. A: Math. Gen. 35, 1297 (2002).

8. A.A. Saharian, The Generalized Abel-Plana Formula with Applications to Bessel Functions and Casimir Effect (Yerevan State University Publishing House, Yerevan, 2008); Preprint ICTP/2007/082; arXiv:0708.1187

9. P. Candelas and D. J. Raine, Phys. Rev. D 12, 965 (1975); J. S. Dowker and R. Critchley, Phys. Rev. D 13, 224 (1976); J. S. Dowker and R. Critchley, Phys. Rev. D 13, 3224 (1976); J. Bros and U. Moschella, Rev. Math. Phys. 8, 327 (1996); R. Bousso, A. Maloney and A. Strominger, Phys. Rev. D 65, 104039 (2002).

10. A. A. Saharian and M. R. Setare, Phys. Lett. B 552, 119 (2003).

11. A. A. Saharian and M. R. Setare, Phys. Lett. B 687, 253 (2010); A. A. Saharian and M. R. Setare, Class. Quantum Grav. 27, 225009 (2010).

12. A. A. Saharian, Phys. Rev. D 69, 085005 (2004).

13. A. A. Saharian and T. A. Vardanyan, in preparation.

14. E. Elizalde, S. D. Odintsov and A. A. Saharian, Phys. Rev. D 79, 065023 (2009).

15. G. Cognola, E. Elizalde, S. Nojiri, S. D. Odintsov and S. Zerbini, J. Cosmol. Astropart. Phys. 02 (2005) 010.

16. I. L. Buchbinder and S. D. Odintsov, Class. Quantum Grav. 2, 721 (1985).

17. A. A. Saharian, Nucl. Phys. B 712, 196 (2005).

18. A. A. Saharian, Phys. Rev. D 73, 044012 (2006); A. A. Saharian, Phys. Rev. D 73, 064019 (2006).

19. P. Breitenlohner and D. Z. Freedman, Ann. Phys. (N. Y.) 144, 249 (1982). 